\title{
Monolimb Paralysis after Laparoscopic Appendectomy Due to Conversion Disorder
}

\author{
Gihyeong Ryu*, Sung Hyuk Song, Kyeong Hwan Lee
}

Department of Rehabilitation Medicine, Dongguk University College of Medicine, Gyeongju, Korea

Limb paralysis can develop for various reasons. We found a 13-year-old patient who became paralyzed in her lower extremities after laparoscopic appendectomy. Some tests, including electrodiagnostic studies and magnetic resonance imaging, were performed to evaluate the cause of lower limb paralysis. None of the tests yielded definite abnormal findings. We subsequently decided to explore the possibility of psychological problems. The patient was treated with simultaneous rehabilitation and psychological counseling. Paralysis of the patient's lower extremity improved gradually and the patient returned to normal life. Our findings indicate that psychological problems can be related to limb paralysis without organ damage in patients who have undergone laparoscopic surgical procedures.

Keywords: Paralysis; Appendectomy; Conversion Disorder

\section{INTRODUCTION}

Limb paralysis can develop for various reasons. The main causes are injury to the nervous system, including the central and peripheral nervous systems, or musculoskeletal abnormalities. In rare cases, paralysis can develop due to psychological problems. In particular, paralysis of the limbs can be caused by a conversion disorder after surgical procedures, including laparoscopic

Received: July 7, 2014, Accepted: October 22, 2014

${ }^{*}$ Corresponding Author: Gihyeong Ryu

Tel: +82-54-770-8294, Fax: +82-54-770-8378

E-mail:mform1003@naver.com

Korean Journal of Family Medicine

Copyright (C) 2014 The Korean Academy of Family Medicine

() This is an open-access article distributed under the terms of the Creative Commons Attribution Non-Commercial License (http://creativecommons.org/licenses/by-nc/3.0) which permits unrestricted noncommercial use, distribution, and reproduction in any medium, provided the original work is properly cited. operations. We found a patient who became paralyzed in her lower extremity after undergoing laparoscopic appendectomy. The patient was treated with rehabilitation and psychological counseling. Our findings indicate that psychological problems can be related to limb paralysis without organ damage in patients who have undergone laparoscopic surgical procedures.

\section{CASE REPORT}

A 13-year-old patient visited the department of general surgery complaining of sudden abdominal pain after experiencing intermittent nausea and vomiting for a few days. Tenderness and rebound tenderness in the right lower portion of her abdomen was observed upon physical examination. Her vital signs were normal. Appendicitis was diagnosed by ultrasonography and a laparoscopic appendectomy was performed. The operation was performed with the patient in a supine position while under general anesthesia. An incision was made $1 \mathrm{~cm}$ below 
the umbilicus, and a trocar $11 \mathrm{~cm}$ in length was inserted after pneumoperitoneum was induced using a Veress needle. Blood vessels were monitored using laparoscopic light transmitted through the abdominal wall. Next, $5 \mathrm{~mm}$ trocars were inserted around the suprapubic area and anti-McBurney's point. The appendix was extracted with laparoscopic forceps and the mesoappendix was removed by laparoscopic electric cautery. Blood vessels were ligated with laparoscopic clips. The base of the appendix was ligated with two Endo-loops (Covidien Co. Ltd., Mansfield, MA, USA) and a $10 \mathrm{~mm}$ distal region of the base was also clipped with a laparoscopic clip. The appendix was resected and removed using a pocket for tissue removal. The laparoscopic appendectomy was completed after intraperitoneal irrigation and bleeding control.

Two days after the operation, the patient complained of hypesthesia and weakness in the right lower extremity. The patient was transferred to the department of rehabilitation medicine, and a neurological examination was performed. The examination revealed that the pathological reflexes and deep tendon reflexes were normal. The ability to sense pain and temperature were normal, but the sense of light touch was abnormal between the T11 and L 2 dermatome, indicative of hypesthesia. Muscle strength of right hip flexor, knee extensor, and knee flexor was reduced to $2 / 5$, but the strength of other muscles was unchanged according to a manual muscle test. Pelvic and lumbar magnetic resonance imaging (MRI) was performed in order to evaluate the cause of lower limb paralysis. None of the tests yielded definite abnormal findings. The patient complained of pain around the surgical site, and paralysis of the right lower extremity persisted for 10 days after surgery. However, there was no pain in the right lower limb. Muscle strength of the right hip flexor, knee extensor, and knee flexor was still decreased in the follow-up manual muscle test. An electrodiagnostic study (EMG) was performed on postoperative day 13. Nerve conduction and needle electromyography results appeared normal, except that the interference pattern of motor unit action potential in the muscles was decreased at maximal volition.

Rehabilitation training was performed by the patient for 40 minutes twice a day with a physical therapist since being transferred to the department of rehabilitation medicine. This procedure included muscle strengthening exercises and functional gait training. The patient indicated that she liked 'taekwondo' and desired to become a taekwondo athlete. Therefore, functional training, including balance and posture exercises related to taekwondo, was performed continuously. No significant improvement of muscle power was observed. However, independent walking was possible although intermittent claudication was present. The sense of balance when in specific positions also improved while functional training was performed.

On postoperative day 14, tenderness around the right lower quadrant of the abdomen was still observed and the degree of the pain was 6 on the visual analogue scale. Because the pain around the operation site and right lower abdomen persisted, a general surgery consultation was performed to evaluate the presence or absence of laparoscopic complications. However, the operation wound was found to be well healed, and no abscesses or mass lesions were found by follow-up abdominal computed tomography. On postoperative day 20 , a follow-up EMG was performed in which no significant interval changes were observed, and the results were the same as the previous EMG. There was no difference between the circumferences of the lower limbs. Therefore, peripheral nerve lesions and myopathy were ruled out as the cause of paralysis. Brain, cervical, and thoracic MRI was performed in order to detect disorders of the central nervous system, but definite abnormal findings were not observed. Daily inspection revealed that there was no significant interval change in muscle weakness. Although limb paralysis persisted, the patient was not worried or depressed. We subsequently decided to explore the possibility of psychological problems after organ damage, including nerve or muscle injury, was ruled out as the cause of paralysis.

An interview with the patient's parents was conducted on postoperative day 21 . According to the results of the interview, the patient was having trouble adjusting to school and her parents had visited the school several times to meet with her teacher. The patient hoped to move to another school that has a taekwondo club in order to be an athlete, but the patient's parents were against the idea. This caused conflict between the patient and her parents.

On postoperative day 22, significant improvement of the leg strength was not observed but the patient could independently walk from the general ward to the rehabilitation clinic. The patient received functional training in the physical therapy department. 
Although right hip flexor strength was still 2/5 the normal level, her lower extremities moved well without claudication from the stance to swing phase during gait training. On postoperative day 23, we interviewed with the patient's parents and explained that a conversion disorder could be a possible cause of paralysis. The parents were encouraged to resolve the conflict concerning the patient's career path through continuous psychiatric consultation. The patient was discharged. We interviewed the patient's parents by telephone a month later. Paralysis of the patient's lower extremity improved gradually and the patient returned to normal life. She has been learning taekwondo after being discharged from the hospital.

\section{DISCUSSION}

Laparoscopic surgery is widely performed instead of open appendectomy due to many advantages, such as diagnostic help, shorter hospitalization periods, reduced post-operative pain, decreased need for analgesics, fewer cases of wound infection, and improved cosmetic results. ${ }^{1)}$ However, direct organ injuries, vascular injury, wound infection, hernia formation, tumor seeding, and endometriosis resulting from laparoscopic surgery have been reported. ${ }^{2)}$ Although rare, paralysis due to nerve injury may also occur owing to positional injury, trocar injury, peritoneal adhesion, or pneumoperitoneum after laparoscopic surgery. ${ }^{3)}$ If organ problems or physical disorders are ruled out as the cause of sensorimotor dysfunction appearing after laparoscopic surgery, a conversion disorder might be the cause of limb paralysis. ${ }^{4,5)}$ We came across a case of paralysis of the lower extremity after laparoscopic appendectomy. Although a complete workup was performed in order to uncover the cause, no organ problems could be found. Therefore, we investigated the possibility that the paralysis may be due to psychological problems. We were aware that the patient was coming into conflict with her parents over the patient's career path and was having trouble adjusting to school. According to the diagnostic criteria of conversion disorder in the DSM-IV, ${ }^{6}$ ) we diagnosed the patient as having conversion disorder due to the motor weakness of the right lower extremity and decision of her career. In addition, there were no organ injuries causing limb paralysis in spite of multilateral evaluations, absences from school due to gait disturbance, and points that were not explained by other symptoms of mental illness.

Conversion disorder is a type of somatoform disorder that causes dyssynergia, tremor, dystonia, or paralysis of one or more extremities. This condition is also known as chronic neurosis, psychosomatic disorder, functional disorder, or functional overlay. ${ }^{7)}$ Conversion disorder is characterized by motor or sensory symptoms accompanied by psychological factors. Functional loss is not intentional or related to general medical problems. ${ }^{8)}$ Conversion disorder must be distinguished from malingering, in which symptoms occur intentionally and are related to secondary gain. One characteristic of conversion disorder is that somatic symptoms are not provoked by intent. ${ }^{9)}$ Ten percent to $23 \%$ of patients with conversion disorder have paralysis of limbs as a major symptom. ${ }^{10)}$ Patients with conversion disorder are more likely amicable, cooperative, and reliant than malingerers and accept evaluation and treatment gladly. Patients with conversion disorder are more likely to report historical gaps, incorrect, and unpredictable changes, whereas malingerers are more likely to provide extremely thorough and definite explanations of their experiences precipitating their illness. ${ }^{11)}$ The patient cooperated well with medical examination and there were vagaries about her symptoms and signs. These factors were helpful in differentiating the diagnosis from malingering.

For patients with paralytic symptoms due to conversion disorder, hospitalization is required for a few day or months, and functional recovery is good. ${ }^{4)}$ Not much research has been conducted on the outcomes or long-term prognosis of conversion disorder in children and adolescents. It has been reported that many conversion disorder symptoms spontaneously improve after a few days or weeks or require minimal intervention. It was also found that the rates of clinical improvement are good, ranging from $56 \%$ to $100 \%$. In particular, a good prognosis is associated with young age, fast diagnosis, rapid identification of stressors, and a good relationship with family members. ${ }^{12)}$ However, it must be noted that the clinical symptoms may be perpetuated if diagnostic tests were performed aggressively in order to identify the causes, but the cause is not detected at an early stage. Symptoms are also more likely to be chronic by excessive concern from family or medical staff. ${ }^{13)}$ In the current study, we only considered the possibility of organic problems soon after the onset of symptoms and thus conducted several diagnostic tests. However, the cause was not identified with these methods and the patient's family 
became increasingly concerned. Fortunately, we determined that the cause was a stressor related to a conflict regarding the patient' $s$ career path. Furthermore, a close relationship was formed between the family members and the parents with respect to the patient's desire to become a 'taekwondo' athlete. This is thought to be an important step as the patient's symptoms were not chronic and completely resolved within 2 months.

Treatments for conversion disorder include group or individual psychotherapy, medications, and rehabilitation therapies. ${ }^{4)}$ Rehabilitation therapy for patients with paralysis generally includes strengthening exercises for the paralytic muscles, stretching exercises to maintain range of motion for each joint, and functional training to improve gait or transfer. It is important to provide this treatment while not showing excessive concern or reinforcing abnormal gait patterns caused by paralysis that is associated with conversion disorder. In particular, Kanarek et al. ${ }^{4)}$ reported that it is important to plan task-oriented goals based on functional training while considering the patient's interests in order to improve the patient's function. Rehabilitation training that included muscle-strengthening exercises and functional gait training was conducted for 40 minutes twice a day with a physical therapist. Functional training, with balance and posture exercises relating to taekwondo, was also performed continuously considering the patient's interest. We believe that a good rapport with the patient through these therapies prevented the development of sequelae associated with conversion disorder and promoted functional improvement.

\section{CONFLICT OF INTEREST}

No potential conflict of interest relevant to this article was reported.

\section{ACKNOWLEDGMENTS}

This work was supported by the Dongguk University Research Fund of 2014. No commercial parties having a direct interest in the results of this research or will provide benefits to the authors or any organization with which the authors are associated.

\section{REFERENCES}

1. Yau KK, Siu WT, Tang CN, Yang GP, Li MK. Laparoscopic versus open appendectomy for complicated appendicitis. J Am Coll Surg 2007;205:60-5.

2. Han NY, Sung DJ, Park BJ, Kim MJ, Cho SB, Kim YH. Imaging of complications associated with port access of abdominal laparoscopic surgery. Abdom Imaging 2014;39:398-410.

3. Porzionato A, Macchi V, Fenato F, Parenti A, De Caro R. Femoral nerve injury after gynecologic laparoscopy. J Minim Invasive Gynecol 2008;15:105-7.

4. Kanarek SL, Stevenson JE, Wakefield H, Reite E, Zumsteg JM, Brockway JA. Inpatient rehabilitation approach for a young woman with conversion hemiparesis and sensory deficits. PM R 2013;5:66-9.

5. Berhane L, Kurman R, Smith S. Lower extremity paralysis after operative laparoscopy from conversion disorder. A case report.J Reprod Med 1998;43:831-5.

6. American Psychiatric Association. Diagnostic and statistical manual of mental disorder: DSM-IV. 4th ed. Washington (DC): American Psychiatric Association; 1994.

7. Tomasson K, Kent D, Coryell W. Somatization and conversion disorders: comorbidity and demographics at presentation. Acta Psychiatr Scand 1991;84:288-93.

8. Nicholson TR, Stone J, Kanaan RA. Conversion disorder: a problematic diagnosis. J Neurol Neurosurg Psychiatry 2011;82:1267-73.

9. Heruti RJ, Levy A, Adunski A, Ohry A. Conversion motor paralysis disorder: overview and rehabilitation model. Spinal Cord 2002;40:327-34.

10. Hafeiz HB. Hysterical conversion: a prognostic study. $\mathrm{Br} \mathrm{J}$ Psychiatry 1980;136:548-51.

11. Sadock BJ, Kaplan HI, Sadock VA. Kaplan \& Sadock's synopsis of psychiatry: behavioral sciences clinical psychiatry. 10th ed. Philadelphia: Lippincott Williams \& Wilkins; 2007.

12. Pehlivanturk B, Unal F. Conversion disorder in children and adolescents: a 4-year follow-up study. J Psychosom Res 2002;52:187-91.

13. Ness D. Physical therapy management for conversion disorder: case series. J Neurol Phys Ther 2007;31:30-9. 